\title{
Impact of Thrombocytopenia on In-Hospital Outcome in Patients Undergoing Percutaneous Coronary Intervention
}

\author{
Zhongxiu Chen $\mathbb{D}^{1},{ }^{1}$ Zheng Liu, ${ }^{2}$ Nan Li, ${ }^{3}$ Ran Liu, ${ }^{3}$ Miye Wang, ${ }^{3}$ Duolao Wang, ${ }^{4}$ Chen Li, ${ }^{1}$ \\ Kai Li, ${ }^{1}$ Fangbo Luo, ${ }^{5}$ and Yong $\mathrm{He} \mathbb{D D}^{1}$ \\ ${ }^{1}$ Department of Cardiology, West China Hospital of Sichuan University, Chengdu, Sichuan, China \\ ${ }^{2}$ Department of Nursing, West China Hospital of Sichuan University, Chengdu, Sichuan, China \\ ${ }^{3}$ Information Center of West China Hospital of Sichuan University, Chengdu, Sichuan, China \\ ${ }^{4}$ Department of Clinical Sciences, Liverpool School of Tropical Medicine, Pembroke Place, Liverpool L3 5QA, UK \\ ${ }^{5}$ Department of Rehabilitation, Community Health Center of Huaxing Wuhou District, Chengdu, Sichuan, China
}

Correspondence should be addressed to Yong He; heyong_huaxi@163.com

Received 22 August 2020; Revised 23 December 2020; Accepted 5 January 2021; Published 13 January 2021

Academic Editor: Ify R. Mordi

Copyright ( 2021 Zhongxiu Chen et al. This is an open access article distributed under the Creative Commons Attribution License, which permits unrestricted use, distribution, and reproduction in any medium, provided the original work is properly cited.

Background. Thrombocytopenia was intuitively considered to be associated with higher risk of bleeding and multiple comorbidities after percutaneous coronary intervention (PCI). However, controversial results exist, and the real-world clinical impact of thrombocytopenia in patients undergoing PCI is largely unknown. The aim of this study was to evaluate the influence of baseline thrombocytopenia on the prognosis of patients undergoing PCI. Methods. Using the West China Hospital Inpatient Sample database, patients who underwent PCI were identified from August 2012 to January 2019. Baseline thrombocytopenia was defined as a preprocedural platelet count of $100 \times 10^{9} / \mathrm{L}$ or less obtained from a routine blood sample taken within 48 hours before coronary PCI. The clinical effect of the advanced thrombocytopenia group $\left(\leq 85 \times 10^{9} / \mathrm{L}\right)$, according to the median value of platelet count in the thrombocytopenia cohort, was further assessed. The primary outcome was a composite of in-hospital death, bleeding events, and post-PCI transfusion. Results. Of 9531 patients enrolled in our study, 936 had baseline thrombocytopenia and 8595 patients did not have. There were no significant differences in the primary outcome between the two groups. However, advanced thrombocytopenia was independently associated with higher risk of primary outcome (OR 1.67, $95 \%$ CI 1.06 to $2.65, p=0.029$ ). Acute coronary syndrome (ACS) patients with thrombocytopenia were associated with higher odds of major bleeding (BARC $\geq 2$ ) (OR 2.56, 95\% CI 1.24 to $5.44, p=0.011$ ). Compared with the nonthrombocytopenia group, the thrombocytopenia group with ticagrelor use had higher odds of major bleeding (OR 9.7, 95\% CI 1.57 to 60.4 versus OR $0.22,95 \%$ CI 0.03 to 1.69 , interaction $p=0.025)$. Conclusions. It seems feasible for patients with thrombocytopenia to receive PCI, but close attention should be paid to advanced thrombocytopenia, the risk of postprocedure bleeding in ACS patients, and the use of more potent $\mathrm{P}_{2} \mathrm{Y}_{12}$ inhibitor.

\section{Introduction}

The management of antiplatelet therapy/percutaneous coronary intervention (PCI) in coronary artery disease (CAD) patients with thrombocytopenia poses a particular challenge for physicians, as they are at higher risk of both bleeding and, paradoxically, ischemic events [1]. The reported incidence of baseline thrombocytopenia was varied approximately from $1 \%$ to $13 \%$ of CAD patients $[2,3]$. However, these patients have almost invariably been excluded from randomized clin- ical trials of PCI or antithrombotic therapies $[4,5]$. No guidelines and only several reports are available on the safety and tolerance of antiplatelet therapy/PCI in CAD patients complicated with thrombocytopenia at baseline, and the results are still controversial. Previous studies revealed that patients with baseline thrombocytopenia had higher risk for bleeding events and death $[3,6]$, while no differences in mortality or bleeding complications between PCI patients with and without thrombocytopenia were also demonstrated $[2,7,8]$. Hence, the real impact of thrombocytopenia in patients 
undergoing PCI is largely unknown. Therefore, this large cohort study was aimed at evaluating the effect of baseline thrombocytopenia on the prognosis of patients undergoing PCI according to the current practice.

\section{Materials and Methods}

2.1. Study Design and Subjects. This retrospective cohort study included 13,920 consecutively enrolled CAD patients with PCI from August 1, 2012, to January 30, 2019, at West China Hospital. The study protocol was approved by the Ethics Committee of West China Hospital of Sichuan University (Sichuan, China). 3733 patients without complete blood count analysis before PCI were excluded. For patients with multiple PCI procedures, only the first PCI in the time period was included, leaving 9531 patients for analysis (Figure 1). For the purpose of this study, baseline thrombocytopenia was defined as a preprocedural platelet count of $100 \times 10^{9} / \mathrm{L}$ or less obtained from a routine blood sample taken within 48 hours before coronary PCI. The thrombocytopenia cohort was further classified into patients with intermediate thrombocytopenia $\left(85 \times 10^{9} / \mathrm{L}<\right.$ platelet count $\left.\leq 100 \times 10^{9} / \mathrm{L}\right)$ and advanced thrombocytopenia $\left(\leq 85 \times 10^{9} / \mathrm{L}\right)$ according to the median value of platelet count of this thrombocytopenia group.

2.2. Data Collection. Clinical data were extracted from the Hospital Information System and obtained through a review of medical records. Baseline characteristics, medical history, comorbidities, and in-hospital management based on prescription records were evaluated by experienced cardiologists. Laboratory test results of enrolled subjects performed in the West China Hospital Biochemistry Laboratory were also collected.

2.3. Clinical Outcomes. The primary outcome was a composite of in-hospital all-cause death, bleeding events, and postPCI transfusion. Secondary outcomes included major adverse cardiovascular events (MACE); a composite of inhospital cardiac mortality, myocardial infarction (MI), target vessel revascularization (TVR), or stent thrombosis; ischemic and hemorrhagic cerebrovascular accident (CVA); and length of stay. The Bleeding Academic Research Consortium (BARC) definition for bleeding was used to describe bleeding events [9]. Major bleeding was categorized as type 2, 3, or 5 (type 2 indicating any overt, actionable sign of bleeding; type 3 indicating bleeding with a decrease in the hemoglobin of $>3 \mathrm{~g}$ per deciliter, any transfusion, cardiac tamponade, or intracranial or ocular involvement; and type 5 indicating fatal bleeding). The MACE endpoints were defined in accordance with the Academic Research Consortium (ARC) definitions [10]. MI was defined according to the third Universal Definition of MI [11]. For PCI-related MI, we used higher biomarker thresholds for confirmation according to the ISCHEMIA trial (International Study of Comparative Health Effectiveness with Medical and Invasive Approaches) [12]. Cardiac mortality was defined as a documented arrhythmogenic death, an unexpected presumed pulseless condition with the absence of an obvious noncardiac explanation, or death due to congestive cardiac failure or structural heart disease. CVA was defined as an acute episode of focal or global neurological dysfunction persisting for more than 24 hours or leaving residual signs and confirmed by skull CT or MRI. Hospital records were searched for all keywords associated with potential bleeding events, including bleeding and transfusion, and a review of medical records. Outcomes were interpreted and judged by an independent cardiologist.

2.4. Statistical Analysis. There were no missing data for the included variables. Some variables with missing data, such as estimated glomerular filtration rate and left ventricular ejection fraction, were not included in our study. All analyses were performed with SPSS version 23 (IBM Corporation, Armonk, NY). Continuous variables were expressed as mean \pm standard deviation or median with $25^{\text {th }}$ and $75^{\text {th }}$ interquartile range and were compared using the Student $t$ -test or Wilcoxon rank-sum test depending on their distributions. Categorical variables were presented as number and percentage and were compared with the $\chi^{2}$ or Fisher exact test. Differences in outcomes were reported as odds ratios (OR) with 95\% confidence intervals (CI). To address the possible imbalance in the participants' characteristics between the test groups, a propensity score approach was used. The propensity score predicting thrombocytopenia was generated using multivariable logistic regression with thrombocytopenia as the dependent variable and fifteen prespecified baseline characteristics and traditional risk factors (age, sex, hypertension, diabetes mellitus, atrial fibrillation, hyperlipidemia, congestive heart failure, chronic kidney disease, chronic liver disease, prior cerebrovascular accident, prior PCI, prior CABG, stents placed $(\geq 2)$, use of intra-aortic balloon pump, and arterial approach) as the independent variables. Logistic regression was performed to identify the association between thrombocytopenia and the risk of clinical outcomes. We used the propensity score as a control variable in the covariateadjusted analysis of the outcome variables [13]. The association between thrombocytopenia and lg10-transformed length of stay (due to lack of normality) was assessed by linear regression analysis. All reported $p$ values were 2-tailed, and $p$ values $<0.05$ were considered statistically significant.

\section{Results}

3.1. Baseline Characteristics. Of 9531 PCI procedures, 936 patients $(9.8 \%)$ had thrombocytopenia at baseline. The median platelet count in patients with thrombocytopenia was $85 \times 10^{9} / \mathrm{L}\left(25\right.$ th, 75 th percentiles, $74 \times 10^{9} / \mathrm{L}, 94 \times 10^{9} /$ L; range, 6 to $\left.100 \times 10^{9} / \mathrm{L}\right)$. All PCI patients used a secondgeneration drug-eluting stent, and approximately $95 \%$ of the stent type was Promus PREMIER ${ }^{\mathrm{TM}}$ (MONORAIL $^{\mathrm{TM}}$, Boston Scientific, USA) and $5 \%$ was GuReater $^{\circledR}$ (CoCr Sirolimus-Eluting Coronary Stent System, LEPU MEDICAL, China). Compared with patients without thrombocytopenia, patients with thrombocytopenia were older, were more commonly men, had higher level of creatinine and total bilirubin, and had a higher prevalence of atrial fibrillation, chronic kidney disease, and chronic liver disease and lesser use of aspirin (Table 1). 


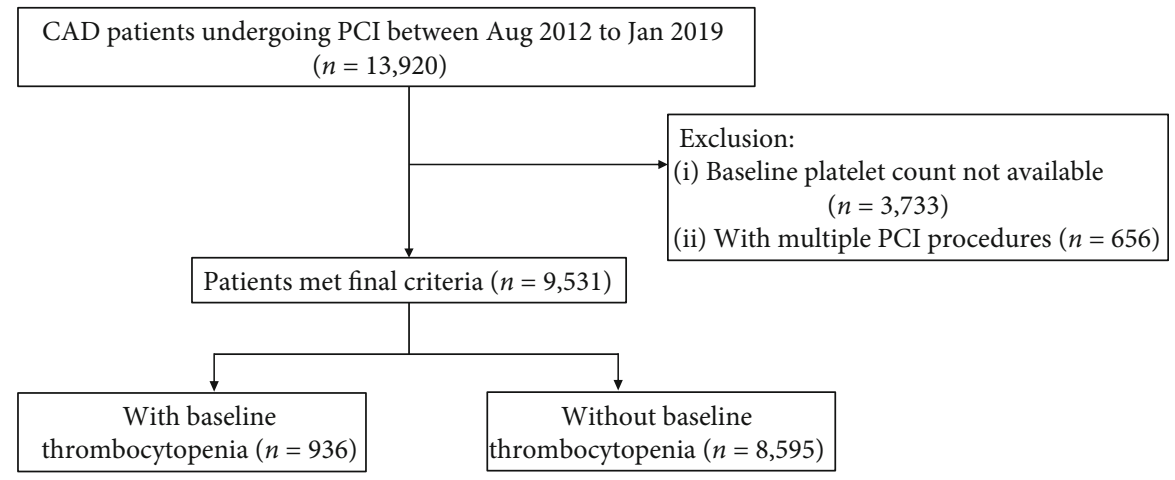

FIGURE 1: Study flowchart describing inclusion and exclusion criteria leading to the final cohort of patients. CAD: coronary artery disease; PCI: percutaneous coronary intervention.

3.2. In-Hospital Clinical Outcomes. Compared with PCI patients without thrombocytopenia, patients with thrombocytopenia had higher prevalence of primary outcome $(4.0 \%$ vs. $2.5 \%, p=0.009)$, including higher prevalence of major bleeding (BARC $\geq 2)(1.3 \%$ vs. $0.6 \%, p=0.014)$ and transfusion $(1.9 \%$ vs. $1.0 \%, p=0.016)$. No significant difference in the secondary outcome was detected between the groups, while the thrombocytopenia cohort had higher prevalence of TVR $(0.3 \%$ vs. $0.1 \%, p=0.042)$. Patients with thrombocytopenia had longer length of hospital stay (5 (3-7) days vs. 4 (3-6) days, $p=0.007)$. After adjusting for the propensity scores in a logistic or linear regression model, thrombocytopenia showed no effects on the rates of primary and secondary outcomes except that thrombocytopenia was associated with higher risk of TVR (OR 4.93, 95\% CI 1.13 to $21.46, p$ $=0.03$ ) (Table 2). Additionally, compared with PCI patients with intermediate thrombocytopenia, the rate of primary outcome, including major bleeding (BARC $\geq 2$ ) and transfusion, was significantly higher in the PCI cohort with advanced thrombocytopenia (Supplemental Table 1). After adjusting for the propensity scores in a logistic regression model, advanced thrombocytopenia was associated with higher risk of primary outcome (OR 1.67, 95\% CI 1.06 to $2.65, p=0.029)$, including higher risk of major bleeding (OR 2.35, 95\% CI 1.11 to $5.00, p=0.027$ ) and transfusion (OR 2.16, 95\% CI 1.17 to $4.00, p=0.014$ ) (Table 3).

3.3. Clinical Outcomes in Patients with Acute Coronary Syndrome. Compared with acute coronary syndrome (ACS) patients without thrombocytopenia, ACS patients with thrombocytopenia had higher prevalence of primary outcome $(5.4 \%$ vs. $3.1 \%, p=0.007)$, including higher prevalence of all-cause mortality $(2.6 \%$ vs. $1.3 \%, 8)$, bleeding $(2.4 \%$ vs. $1.2 \%, p=0.024)$, major bleeding (BARC $\geq 2)(2.2 \%$ vs. $0.7 \%, p=0.001)$, and transfusion ( $2.6 \%$ vs. $1.4 \%, p=0.027)$. No significant difference in the secondary outcome was detected between the groups (Table 4). After adjusting for the propensity scores, ACS patients with thrombocytopenia were only associated with higher risk of major bleeding $(\mathrm{BARC} \geq 2)(\mathrm{OR} 2.56,95 \%$ CI 1.24 to $5.44, p=0.011)$, while there were no effects on the rates of other in-hospital outcomes. There were no significant differences in clinical outcomes in the chronic coronary syndrome cohort with or without thrombocytopenia (Supplemental Table 2).

3.4. Clinical Outcomes Stratified by Usage of Ticagrelor. Specifically, among patients who received more potent $\mathrm{P} 2 \mathrm{Y}_{12}$ inhibitor (ticagrelor), we determined if there was variability in ticagrelor-associated outcomes in PCI patients with thrombocytopenia. As shown in Figure 2, there were no significant differences in the treatment effect of ticagrelor in rates of primary outcome in patients with thrombocytopenia (OR 2.10, 95\% CI 0.50 to 8.83 ) or without thrombocytopenia (OR $1.28,95 \%$ CI 0.74 to 2.23 , interaction $p=0.83$ ). Likewise, no statistical evidence of heterogeneity was found between the thrombocytopenia and nonthrombocytopenia cohorts for the secondary outcome. However, compared with the nonthrombocytopenia group, we did observe higher odds of bleeding (OR 6.48, 95\% CI 1.12 to 37.66 versus OR 0.92 , $95 \%$ CI 0.36 to 2.38 , interaction $p=0.08$ ) and major bleeding (OR 9.7, 95\% CI 1.57 to 60.4 versus OR 0.22 , 95\% CI 0.03 to 1.69 , interaction $p=0.025$ ) in the thrombocytopenia group with ticagrelor use.

\section{Discussion}

Several important findings were observed in this large retrospective study of contemporary domestic real-world clinical practices of PCI and periprocedural antithrombotic use among $9531 \mathrm{CAD}$ patients from over approximately 8 years from 2012 to 2019. Firstly, the incidence of baseline thrombocytopenia in patients undergoing PCI was common, occurring in approximately one in ten patients. Secondly, although there is high prevalence of baseline thrombocytopenia, the incidence of in-hospital adverse events did not increase significantly in the thrombocytopenia group when compared with the normal platelet group. However, advanced thrombocytopenia was independently associated with higher risk of the primary outcome. Thirdly, thrombocytopenia in the ACS subgroup was associated with higher risk of in-hospital major bleeding, while there were no effects on the rates of all-cause mortality, cardiac mortality, CVA, and transfusion and on the length of hospital stay. Fourthly, higher risks of bleeding or major bleeding were observed when ticagrelor was used in thrombocytopenia patients. 
TABle 1: Demographic and clinical characteristics of patients with and without thrombocytopenia.

\begin{tabular}{|c|c|c|c|}
\hline Variable & With TCP $(n=936)$ & Without TCP $(n=8595)$ & $p$ value \\
\hline Mean age (yrs) & $67.1 \pm 10.2$ & $64.6 \pm 11.5$ & $<0.001$ \\
\hline Male, $n(\%)$ & $758(81.0)$ & $6614(77.0)$ & 0.005 \\
\hline Platelet $\left(\times 10^{9} / \mathrm{L}\right)$ & $85(74-94)$ & $174(141-215)$ & $<0.001$ \\
\hline Creatinine $(\mu \mathrm{mol} / \mathrm{L})$ & $85(72-103)$ & $81(69-95)$ & $<0.001$ \\
\hline Total bilirubin $(\mu \mathrm{mol} / \mathrm{L})$ & $13.1(9.8-17.2)$ & $10.9(8.0-14.8)$ & $<0.001$ \\
\hline Hypertension, $n(\%)$ & $514(54.9)$ & $4949(57.6)$ & 0.12 \\
\hline Diabetes mellitus, $n(\%)$ & $273(29.2)$ & $2409(28.0)$ & 0.46 \\
\hline Atrial fibrillation, $n(\%)$ & $54(5.8)$ & $332(3.9)$ & 0.005 \\
\hline Hyperlipidemia, $n$ (\%) & $68(7.2)$ & $714(8.3)$ & 0.27 \\
\hline Chronic kidney disease, $n(\%)$ & $55(5.9)$ & $316(3.7)$ & 0.001 \\
\hline Chronic liver disease, $n(\%)$ & $18(1.9)$ & $44(0.5)$ & $<0.001$ \\
\hline Unstable angina, $n(\%)$ & $265(28.3)$ & $2330(27.1)$ & 0.43 \\
\hline NSTEMI, $n(\%)$ & $92(9.8)$ & $813(9.5)$ & 0.71 \\
\hline STEMI, $n(\%)$ & $140(15.0)$ & $1445(16.8)$ & 0.15 \\
\hline Cardiogenic shock, $n$ (\%) & $23(2.5)$ & $145(1.7)$ & 0.09 \\
\hline Congestive heart failure, $n(\%)$ & $112(12.0)$ & $856(10.0)$ & 0.054 \\
\hline Prior cerebrovascular accident, $n(\%)$ & $21(2.2)$ & $234(2.8)$ & 0.39 \\
\hline Prior PCI, $n(\%)$ & $125(13.4)$ & $1124(13.1)$ & 0.81 \\
\hline Prior CABG, $n(\%)$ & $8(0.9)$ & $53(0.6)$ & 0.39 \\
\hline IABP, $n(\%)$ & $53(5.7)$ & $397(4.6)$ & 0.15 \\
\hline Stents placed $(\geq 2), n(\%)$ & $278(29.7)$ & $2329(27.1)$ & 0.09 \\
\hline Arterial approach & & & 0.27 \\
\hline Radial, $n(\%)$ & $843(90.1)$ & $7844(91.3)$ & \\
\hline Femoral, $n(\%)$ & $47(5.0)$ & $407(4.7)$ & \\
\hline Both, $n(\%)$ & $36(3.8)$ & $237(2.8)$ & \\
\hline Other, $n(\%)$ & $10(1.1)$ & $107(1.2)$ & \\
\hline \multicolumn{4}{|l|}{ Periprocedural medicine } \\
\hline Aspirin, $n(\%)$ & $903(96.5)$ & $8449(98.3)$ & $<0.001$ \\
\hline Clopidogrel, $n(\%)$ & 907 (96.9) & $8321(96.8)$ & 0.88 \\
\hline Ticagrelor, $n(\%)$ & $41(4.4)$ & $410(4.8)$ & 0.59 \\
\hline Warfarin, $n(\%)$ & $12(1.3)$ & $116(1.3)$ & 0.87 \\
\hline NOAC, $n(\%)$ & $3(0.3)$ & $27(0.3)$ & 1.0 \\
\hline Tirofiban, $n(\%)$ & $161(17.2)$ & $1531(17.8)$ & 0.64 \\
\hline Enoxaparin, $n(\%)$ & $254(27.1)$ & $2448(28.5)$ & 0.39 \\
\hline
\end{tabular}

TCP: thrombocytopenia; LDL-C: low-density lipoprotein cholesterol; NSTEMI: non-ST-segment elevation myocardial infarction; STEMI: ST-segment elevation myocardial infarction; PCI: percutaneous coronary intervention; CABG: coronary artery bypass graft; IABP: intra-aortic balloon pump; NOAC: new oral anticoagulants.

Our results are consistent with Raphael et al.'s [8] and Liu et al.'s [2] findings. In Raphael et al.'s retrospective analysis (thrombocytopenia was defined as platelet count $<100 \times 10^{9} /$ L, $n=204$ ), in-hospital bleeding events and death after PCI were similar in patients with and without thrombocytopenia. Although a higher number of bleeding events in the thrombocytopenia group were detected on follow-up, this phenomenon happened at 5 years after PCI and was largely gastrointestinal in nature, unrelated to the PCI procedure and dual antiplatelet therapy. Liu et al.'s study was aimed at investigating the longterm impact of thrombocytopenia (defined as platelet count $\left.<150 \times 10^{9} / \mathrm{L}, n=1263\right)$ in Chinese patients undergoing elective PCI. No differences in 30-month adverse outcomes, including mortality and bleeding complications, were detected between patients with and without thrombocytopenia. Additionally, in cancer patients with ACS and chronic thrombocytopenia, no procedure- or antiplatelet therapy-related cerebrovascular events were noted. Moderate thrombocytopenia was associated with decreased overall survival, whereas aspirin, dual antiplatelet therapy, and statin use showed a trend of improved overall survival [14]. These results indicated that it seemed feasible for patients with moderate chronic thrombocytopenia to receive PCI as well as guideline-recommended duration of antiplatelet therapy. 
TABLE 2: The association between thrombocytopenia and the occurrence of clinical outcomes.

\begin{tabular}{|c|c|c|c|c|c|}
\hline Clinical outcomes & $\begin{array}{l}\text { With TCP } \\
(n=936)\end{array}$ & $\begin{array}{l}\text { Without TCP } \\
\quad(n=8595) \\
n(\%)\end{array}$ & $\begin{array}{c}p \\
\text { value }\end{array}$ & $\begin{array}{l}\text { Adjusted odds ratio (95\% } \\
\text { CI) }\end{array}$ & $\begin{array}{c}p \\
\text { value }\end{array}$ \\
\hline $\begin{array}{l}\text { Primary outcome: all-cause mortality, bleeding, or } \\
\text { transfusion }\end{array}$ & $37(4.0)$ & $215(2.5)$ & 0.009 & $1.30(0.88-1.93)$ & 0.19 \\
\hline All-cause mortality & $16(1.7)$ & $93(1.1)$ & 0.09 & $1.23(0.67-2.25)$ & 0.50 \\
\hline Bleeding & $14(1.5)$ & $85(1.0)$ & 0.15 & & \\
\hline $\mathrm{BARC} \geq 2$ & $12(1.3)$ & $51(0.6)$ & 0.014 & $1.90(0.98-3.68)$ & 0.06 \\
\hline BARC 1 & $2(0.2)$ & $34(0.4)$ & & & \\
\hline BARC 2 & $6(0.6)$ & $21(0.2)$ & & & \\
\hline BARC 3a & $4(0.4)$ & $16(0.2)$ & & & \\
\hline BARC 3b & $1(0.1)$ & $9(0.1)$ & & & \\
\hline BARC $3 c$ & $0(0)$ & $3(0)$ & & & \\
\hline BARC 5a & $1(0.1)$ & $2(0)$ & & & \\
\hline Transfusion & $18(1.9)$ & $90(1.0)$ & 0.016 & $1.53(0.88-2.66)$ & 0.13 \\
\hline RBC transfusion & $16(1.7)$ & $79(0.9)$ & 0.021 & $1.52(0.85-2.72)$ & 0.16 \\
\hline Platelet transfusion & $1(0.1)$ & $4(0.05)$ & 0.99 & & \\
\hline Plasma transfusion & $4(0.4)$ & $18(0.2)$ & 0.34 & & \\
\hline Secondary outcome: MACE or CVA & $28(3.0)$ & $212(2.5)$ & 0.33 & $1.03(0.68-1.57)$ & 0.89 \\
\hline MACE & $19(2.0)$ & $123(1.4)$ & 0.15 & $1.14(0.67-1.94)$ & 0.63 \\
\hline Cardiac mortality & $13(1.4)$ & $85(1.0)$ & 0.25 & $1.08(0.56-2.06)$ & 0.82 \\
\hline Myocardial infarction & $3(0.3)$ & $29(0.3)$ & 1.0 & & \\
\hline Target vessel revascularization & $3(0.3)$ & $5(0.1)$ & 0.042 & $4.93(1.13-21.46)$ & 0.03 \\
\hline Stent thrombosis & $0(0)$ & $8(0.1)$ & 1.0 & & \\
\hline Ischemic CVA & $9(1.0)$ & $93(1.1)$ & 0.73 & $0.83(0.41-1.65)$ & 0.59 \\
\hline Hemorrhagic CVA & $0(0)$ & $3(0)$ & 1.0 & & \\
\hline Length of stay (days) & $5(3-7)$ & $4(3-6)$ & 0.007 & $0.01(-0.01-0.02)^{\&}$ & 0.52 \\
\hline
\end{tabular}

PCI: percutaneous coronary intervention; TCP: thrombocytopenia; CI: confidence interval; BARC: Bleeding Academic Research Consortium; RBC: red blood cell; MACE: major adverse cardiovascular events, including in-hospital cardiac mortality, myocardial infarction, target vessel revascularization, or stent thrombosis; CVA: cerebrovascular accident. ${ }^{\$}$ Adjusted for the propensity score in regression models. ${ }^{8} \beta$ coefficient $(95 \% \mathrm{CI})$ of lg10-transformed length of stay.

TABLE 3: The association between advanced thrombocytopenia (platelet count $\leq 85 \times 10^{9} / \mathrm{L}$ ) and the occurrence of clinical outcomes.

\begin{tabular}{lcc}
\hline Clinical outcomes & Adjusted odds ratio (95\% CI) & $p$ value \\
\hline Primary outcome & $1.67(1.06-2.65)$ & 0.029 \\
All-cause mortality & $1.17(0.56-2.45)$ & 0.68 \\
Bleeding & $1.38(0.67-2.87)$ & 0.384 \\
BARC $\geq 2$ & $2.35(1.11-5.00)$ & 0.027 \\
Transfusion & $2.16(1.17-4.00)$ & 0.014 \\
Secondary outcome & $1.27(0.76-2.12)$ & 0.366 \\
\hline
\end{tabular}

CI: confidence interval; BARC: Bleeding Academic Research Consortium.

Controversially, some studies demonstrated opposite conclusion. Ito et al. [3] evaluated the influence of thrombocytopenia (defined as platelet count $<150 \times 10^{9} / \mathrm{L}$ ) on PCI patients in the pooled database from 3 prospective studies in Japan, CREDO- (Coronary Revascularization Demonstrating Outcome-) Kyoto PCI/CABG registry cohort-2, RESET (the Randomized Evaluation of Sirolimus-Eluting Versus Everolimus-Eluting Stent Trial), and NEXT
(NOBORI Biolimus-Eluting Versus XIENCE/PROMUS Everolimus-Eluting Stent Trial), and demonstrated that even mild thrombocytopenia at baseline was associated with higher risk for bleeding events and all-cause death through a 3-year follow-up (both within and beyond 30 days). Yadav et al. [15] reported that baseline thrombocytopenia was not associated with the crude event at 30 days but represented a hematologic marker of poor prognosis and was strongly associated with adverse ischemic events 1 year after PCI in pooled populations from 2 large-scale randomized trials: the ACUITY (Acute Catheterization and Urgent Intervention Triage Strategy) [16] and HORIZONS-AMI (Harmonizing Outcomes With Revascularization and Stents in Acute Myocardial Infarction) [17] trials. More recently, Ayoub et al.'s study [6] identified patients who underwent PCI with or without chronic thrombocytopenia from the National Inpatient Sample database of the UK and revealed that patients with chronic thrombocytopenia were at higher risk of in-hospital mortality (OR 2.30, 95\% CI 1.90 to $2.70, p<$ 0.0001 ) and bleeding complications (OR 2.40, 95\% CI 2.05 to $2.72, p<0.0001$ ).

The contrary scenario can be attributed to the different cut-off values of platelet count for thrombocytopenia, study 
TABLE 4: The association between thrombocytopenia and the occurrence of in-hospital outcomes in the ACS cohort ( $n=5078)$.

\begin{tabular}{|c|c|c|c|c|c|}
\hline \multirow[b]{2}{*}{ Clinical outcomes } & \multicolumn{5}{|c|}{ ACS cohort $(n=5078)$} \\
\hline & $\begin{array}{l}\text { With TCP } \\
(n=497)\end{array}$ & $\begin{array}{l}\text { Without TCP } \\
\quad(n=4581) \\
n(\%)\end{array}$ & $\begin{array}{c}p \\
\text { value }\end{array}$ & $\begin{array}{l}\text { Adjusted odds ratio }(95 \% \\
\qquad \mathrm{CI})^{\$}\end{array}$ & $\begin{array}{c}p \\
\text { value }\end{array}$ \\
\hline $\begin{array}{l}\text { Primary outcome: all-cause mortality, bleeding, or } \\
\text { transfusion }\end{array}$ & $27(5.4)$ & $143(3.1)$ & 0.007 & $1.33(0.82-2.16)$ & 0.25 \\
\hline All-cause mortality & $13(2.6)$ & $59(1.3)$ & 0.017 & $1.44(0.71-2.93)$ & 0.31 \\
\hline Bleeding & $12(2.4)$ & $55(1.2)$ & 0.024 & $1.71(0.88-1.71)$ & 0.12 \\
\hline $\mathrm{BARC} \geq 2$ & $11(2.2)$ & $31(0.7)$ & 0.001 & $2.56(1.24-5.44)$ & 0.011 \\
\hline Transfusion & $13(2.6)$ & $62(1.4)$ & 0.027 & $1.44(0.74-2.82)$ & 0.28 \\
\hline $\mathrm{RBC}$ transfusion & $13(2.6)$ & $53(1.6)$ & 0.006 & $1.74(0.88-3.44)$ & 0.11 \\
\hline Platelet transfusion & $1(0.2)$ & $2(0)$ & 0.69 & & \\
\hline Plasma transfusion & $3(0.6)$ & $11(0.2)$ & 0.31 & & \\
\hline Secondary outcome: MACE or CVA & $19(3.8)$ & $123(2.7)$ & 0.14 & $1.10(0.65-1.86)$ & 0.73 \\
\hline MACE & $15(3.0)$ & $75(1.6)$ & 0.027 & $1.34(0.71-2.53)$ & 0.36 \\
\hline Cardiac mortality & $11(2.2)$ & $55(1.2)$ & 0.058 & $1.37(0.65-2.90)$ & 0.41 \\
\hline Myocardial infarction & $3(0.6)$ & $18(0.4)$ & 0.74 & & \\
\hline Target vessel revascularization & $1(0.2)$ & $2(0)$ & 0.69 & & \\
\hline Stent thrombosis & $0(0)$ & $4(0.1)$ & 1.0 & & \\
\hline Ischemic CVA & $4(0.8)$ & $53(1.2)$ & 0.63 & & \\
\hline Hemorrhagic CVA & $0(0)$ & $1(0)$ & 1.0 & & \\
\hline Length of stay (days) & $5(3-7)$ & $4(3-7)$ & 0.017 & $0.01(-0.01-0.02)^{\&}$ & 0.56 \\
\hline
\end{tabular}

ACS: acute coronary syndrome; TCP: thrombocytopenia; CI: confidence interval; MACE: major adverse cardiovascular events, including in-hospital cardiac mortality, myocardial infarction, target vessel revascularization, or stent thrombosis; CVA: cerebrovascular accident; BARC: Bleeding Academic Research Consortium; RBC: red blood cell. ${ }^{\$}$ Adjusted for the propensity score in regression models. ${ }^{\star} \beta$ coefficient $(95 \%$ CI) of lg10-transformed length of stay.

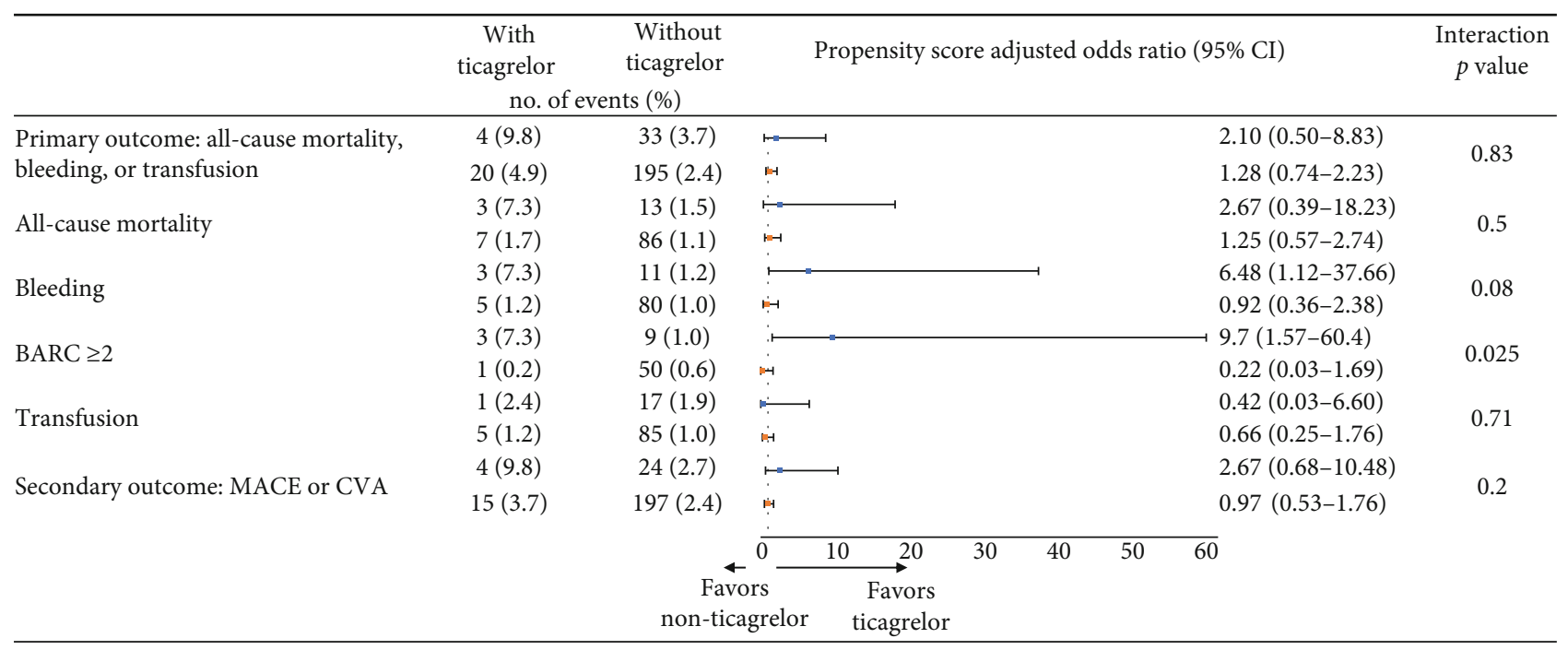

- TCP cohort $(n=936)$

non-TCP cohort $(n=8595)$

FIGURE 2: The association between ticagrelor use and the risk of in-hospital outcomes in patients with and without TCP. TCP: thrombocytopenia; BARC: Bleeding Academic Research Consortium; MACE: major adverse cardiovascular events; CVA: cerebrovascular accident.

population, disease severity, definitions of bleeding criteria, PCI strategy and antithrombotic regimen, the exclusion of patients with severe thrombocytopenia from some studies, and varying follow-up durations. For example, in Ito et al.'s pooled study of 3 different era researches [3], bare-metal stent, the first-generation DES, or older antiplatelet agents (ticlopidine) were used in a large proportion of patients (different from the current PCI practice), and bleeding was 
defined in the Global Utilization of Streptokinase and Tissue Plasminogen Activator for Occluded arteries trial (GUSTO). ACUITY and HORIZONS-AMI trials recruited patients with acute coronary syndromes, excluded all patients with platelet counts $<100 \times 10^{9} / \mathrm{L}$, had more severe disease in the thrombocytopenia group $\left(100-150 \times 10^{9} / \mathrm{L}\right)$, and routinely used glycoprotein IIb/IIIa inhibitor, which is selective for bailout use according to the current practice. In our cohort, we did observe higher bleeding risk with usage of tirofiban in both thrombocytopenia and nonthrombocytopenia cohorts, while it is more obvious in the thrombocytopenia cohort (Supplemental Figure 1), which furtherly confirmed the higher bleeding effect of the combined use of glycoprotein IIb/IIIa inhibitor.

In Ayoub et al.'s [6] large cohort study, chronic thrombocytopenia patients (durations of $>1$ year) were identified by administrative code at discharge from a claim-based database, not validated by single patients' blood counts. Thus, the degree of thrombocytopenia might be more severe and more caused by malignancy or chronic liver disease and associated with poorer outcomes. Moreover, studies reported that the association between platelet count and long-term mortality functioned in a U-shaped fashion [18], with both lower and higher platelet counts associated with a greater mortality risk. Ito et al.'s study [3] confirmed the U-shaped model. The retrospective analysis from the Mayo Clinic revealed that a platelet count of less than $50 \times 10^{9} / \mathrm{L}$ was considered more significantly increased bleeding risk [8]. Consistently, in our study, after adjusting for the propensity scores in a logistic regression model, we found that advanced thrombocytopenia was associated with higher risk of the primary outcome. Thus, special attention should be paid to PCI patients with advanced/severe thrombocytopenia.

Recently, the Academic Research Consortium for High Bleeding Risk (ARC-HBR) reported a standardized definition of HBR (a predicted annual BARC 3 or 5 bleeding rate of $\geq 4 \%$ or an intracranial hemorrhage risk of $\geq 1 \%$ ) in patients undergoing PCI [19]. Anticipated use of longterm oral anticoagulation, severe or end-stage chronic kidney disease $(e G F R<30 \mathrm{~mL} / \mathrm{min})$, hemoglobin $<11 \mathrm{~g} / \mathrm{dL}$, moderate or severe baseline thrombocytopenia (platelet count $<100 \times 109 / \mathrm{L}$ ), and chronic bleeding diathesis are considered important factors of HBR. Moreover, low body weight, frailty, heart failure, and dialysis are listed as risk factors of the HBR criteria for Asian patients [20]. Our study showed that baseline thrombocytopenia did not appear to have a clinically significant effect on in-hospital adverse outcomes among patients who underwent PCI, while the risk of bleeding was increased in the ACS subgroup with thrombocytopenia and in thrombocytopenia patients with ticagrelor. Given the demonstrated link between bleeding and increased mortality after PCI $[21,22]$, it is important for clinicians to pay close attention to the risk of postprocedure bleeding in ACS patients and cautiously use ticagrelor because of its higher bleeding risk [1, 23, 24]. In our study, a total of 3 patients had hemorrhagic CVA and all were in the nonthrombocytopenia cohort. That means, besides platelet number, clinicians should also pay attention to the function of platelets and take other bleeding risk factors, such as other major and minor HBR criteria, into account comprehensively, and recommended methods for minimizing bleeding risk, including favoring the radial access, careful dosing of antithrombotic agents, and prescribing a proton pump inhibitor, should be taken in high bleeding risk patients. Similar to Eikelboom et al.'s [25] and Yadav et al.'s [15] observation, other used antithrombotic agent did not have a significant effect on the association between baseline thrombocytopenia and in-hospital adverse clinical events in our study. This reminds us that baseline thrombocytopenia is most probably not a mediator of mortality in most cases, and less intensive antithrombotic during or after PCI in patients with baseline thrombocytopenia may lead to the increase in ischemic events. The higher odds of TVR in the thrombocytopenia group of our study may be attributed to its lesser periprocedural prescription of aspirin. Therefore, we should assess comprehensively the severity of the atherothrombotic event and the overall clinical status for prognosis prediction.

4.1. Limitations. This study has several limitations. Firstly, despite the use of the propensity score approach for controlling for possible confounders, the observed results may still subject to imbalances between patients with or without thrombocytopenia due to unmeasured confounders, such as clotting of platelet when collecting blood samples occurring sometimes in the clinical settings, which may mislead to thrombocytopenia. Secondly, the causes of baseline thrombocytopenia were not investigated in every case, and heparin or antiplatelet drug administration prehospital could potentially influence platelet counts. However, the hazard of thrombocytopenia was adjusted with the presentation of acute myocardial infarction and previous PCI, CABG, and stroke, which is linked with antiplatelet therapy. Thirdly, the sample of PCI patients with ticagrelor was relatively small because ticagrelor only became available in China in recent years. Prospective randomized studies are needed to provide robust data. Lastly, we did not explore the long-term effect of baseline thrombocytopenia, and only nine patients had severe thrombocytopenia in our study. Thus, future prospective studies with larger sample size and more patients with severe thrombocytopenia are required.

\section{Conclusions}

Although baseline thrombocytopenia was common among patients who underwent PCI, it did not appear to have a clinically significant effect on in-hospital adverse outcomes. However, adverse events were increased in patients with advanced thrombocytopenia. In addition, bleeding risk was increased in the ACS subgroup with thrombocytopenia and was significantly higher in thrombocytopenia patients with ticagrelor. This retrospective single-center study indicated that it seemed feasible for patients with intermediate thrombocytopenia to receive PCI as well as conventional antiplatelet therapy. Clinicians should pay close attention to the advanced thrombocytopenia cohort and the risk of 
postprocedure bleeding in ACS patients and cautiously use potent $\mathrm{P}_{2} \mathrm{Y}_{12}$ inhibitor, such as ticagrelor.

\section{Data Availability}

The data underlying the findings of the paper are freely available on request through the authors themselves. Yong $\mathrm{He}$ (Department of Cardiology, West China Hospital of Sichuan University, 37 Guo Xue Xiang, Chengdu, Sichuan, 610041, China; e-mail: heyong_huaxi@163.com) should be contacted to request the data.

\section{Disclosure}

The funders had no role in the study design, data collection and analysis, decision to publish, or preparation of the manuscript.

\section{Conflicts of Interest}

The authors declare that they have no conflicts of interest.

\section{Authors' Contributions}

Zhongxiu Chen and Zheng Liu contributed equally to this work.

\section{Acknowledgments}

This study was funded by the grant from the Key Research and Development Programs of Sichuan Province (grant numbers 2020YFS0244 and 2020YFS0242).

\section{Supplementary Materials}

Supplemental figure 1. The association between tirofiban using and the risk of in-hospital outcomes in patients with and without TCP. TCP, thrombocytopenia; BARC, Bleeding Academic Research Consortium; MACE, major adverse cardiovascular events; CVA, cerebrovascular accident. Supplemental Table 1. The occurrence of clinical outcomes among PCI cohort without TCP, and with intermediate or advanced thrombocytopenia. Supplemental Table 2. In-hospital outcomes in CCS patients with and without TCP $(\mathrm{n}=4453)$. (Supplementary materials)

\section{References}

[1] C. P. McCarthy, G. Steg, and D. L. Bhatt, "The management of antiplatelet therapy in acute coronary syndrome patients with thrombocytopenia: a clinical conundrum," European Heart Journal, vol. 38, no. 47, pp. 3488-3492, 2017.

[2] S. Liu, C. Song, Y. Zhao et al., "Impact of baseline thrombocytopenia on the long-term outcome of patients undergoing elective percutaneous coronary intervention: an analysis of 9, 897 consecutive patients," Catheterization and Cardiovascular Interventions, vol. 93, no. S1, pp. 764-771, 2019.

[3] S. Ito, H. Watanabe, T. Morimoto et al., "Impact of baseline thrombocytopenia on bleeding and mortality after percutaneous coronary intervention," The American Journal of Cardiology, vol. 121, no. 11, pp. 1304-1314, 2018.
[4] S. James, A. Åkerblom, C. P. Cannon et al., "Comparison of ticagrelor, the first reversible oral $\mathrm{P}_{2} \mathrm{Y}_{12}$ receptor antagonist, with clopidogrel in patients with acute coronary syndromes: Rationale, design, and baseline characteristics of the PLATelet inhibition and patient Outcomes (PLATO) trial," American Heart Journal, vol. 157, no. 4, pp. 599-605, 2009.

[5] D. L. Bhatt, G. W. Stone, K. W. Mahaffey et al., "Effect of platelet inhibition with cangrelor during PCI on ischemic events," The New England Journal of Medicine, vol. 368, no. 14, pp. 1303-1313, 2013.

[6] K. Ayoub, M. Marji, G. Ogunbayo et al., "Impact of chronic thrombocytopenia on in-hospital outcomes after percutaneous coronary intervention," JACC. Cardiovascular Interventions, vol. 11, no. 18, pp. 1862-1868, 2018.

[7] J. Shiraishi, N. Koshi, Y. Matsubara et al., "Effects of baseline thrombocytopenia on in-hospital outcomes in patients undergoing elective percutaneous coronary intervention," Internal Medicine, vol. 58, no. 12, pp. 1681-1688, 2019.

[8] C. E. Raphael, D. B. Spoon, M. R. Bell et al., "Effect of preprocedural thrombocytopenia on prognosis after percutaneous coronary intervention," Mayo Clinic Proceedings, vol. 91, no. 8, pp. 1035-1044, 2016.

[9] R. Mehran, S. V. Rao, D. L. Bhatt et al., "Standardized bleeding definitions for cardiovascular clinical trials: a consensus report from the bleeding academic research consortium," Circulation, vol. 123, no. 23, pp. 2736-2747, 2011.

[10] D. E. Cutlip, S. Windecker, R. Mehran et al., "Clinical end points in coronary stent trials: a case for standardized definitions," Circulation, vol. 115, no. 17, pp. 2344-2351, 2007.

[11] K. Thygesen, J. S. Alpert, A. S. Jaffe et al., "Third universal definition of myocardial infarction," Journal of the American College of Cardiology, vol. 60, no. 16, pp. 1581-1598, 2012.

[12] D. J. Maron, J. S. Hochman, H. R. Reynolds et al., "Initial invasive or conservative strategy for stable coronary disease," The New England Journal of Medicine, vol. 382, no. 15, pp. 1395$1407,2020$.

[13] L. Q. Yue, "Statistical and regulatory issues with the application of propensity score analysis to nonrandomized medical device clinical studies," Journal of Biopharmaceutical Statistics, vol. 17, no. 1, pp. 1-13, 2007.

[14] C. Iliescu, D. V. Balanescu, T. Donisan et al., "Safety of diagnostic and therapeutic cardiac catheterization in cancer patients with acute coronary syndrome and chronic thrombocytopenia," The American Journal of Cardiology, vol. 122, no. 9, pp. 1465-1470, 2018.

[15] M. Yadav, P. Généreux, G. Giustino et al., "Effect of baseline thrombocytopenia on ischemic outcomes in patients with acute coronary syndromes who undergo percutaneous coronary intervention," The Canadian Journal of Cardiology, vol. 32, no. 2, pp. 226-233, 2016.

[16] G. W. Stone, B. T. McLaurin, D. A. Cox et al., "Bivalirudin for patients with acute coronary syndromes," New England Journal of Medicine, vol. 355, no. 21, pp. 2203-2216, 2006.

[17] G. W. Stone, B. Witzenbichler, G. Guagliumi et al., "Bivalirudin during primary PCI in acute myocardial infarction," The New England Journal of Medicine, vol. 358, no. 21, pp. 2218 2230, 2008.

[18] P. Msaouel, A. P. Lam, K. Gundabolu et al., "Abnormal platelet count is an independent predictor of mortality in the elderly and is influenced by ethnicity," Haematologica, vol. 99, no. 5, pp. 930-936, 2014. 
[19] P. Urban, R. Mehran, R. Colleran et al., "Defining high bleeding risk in patients undergoing percutaneous coronary intervention," Circulation, vol. 140, no. 3, pp. 240-261, 2019.

[20] M. Nakamura, K. Kimura, T. Kimura et al., "JCS 2020 guideline focused update on antithrombotic therapy in patients with coronary artery disease," Circulation Journal, vol. 84 , no. 5, pp. 831-865, 2020.

[21] P. G. Steg, K. Huber, F. Andreotti et al., "Bleeding in acute coronary syndromes and percutaneous coronary interventions: position paper by the Working Group on Thrombosis of the European Society of Cardiology," European Heart Journal, vol. 32, no. 15, pp. 1854-1864, 2011.

[22] R. Mehran, S. Pocock, E. Nikolsky et al., "Impact of bleeding on mortality after percutaneous coronary intervention results from a patient-level pooled analysis of the REPLACE-2 (Randomized Evaluation of PCI Linking Angiomax to Reduced Clinical Events), ACUITY (Acute Catheterization and Urgent Intervention Triage Strategy), and HORIZONS-AMI (Harmonizing Outcomes with Revascularization and Stents in Acute Myocardial Infarction) trials," JACC. Cardiovascular Interventions, vol. 4, no. 6, pp. 654-664, 2011.

[23] M. Valgimigli, H. Bueno, R. A. Byrne et al., "2017 ESC focused update on dual antiplatelet therapy in coronary artery disease developed in collaboration with EACTS: the Task Force for dual antiplatelet therapy in coronary artery disease of the European Society of Cardiology (ESC) and of the European Association for Cardio-Thoracic Surgery (EACTS)," European Heart Journal, vol. 39, no. 3, pp. 213-260, 2018.

[24] D. L. Bhatt, J. S. Hulot, D. J. Moliterno, and R. A. Harrington, "Antiplatelet and anticoagulation therapy for acute coronary syndromes," Circulation Research, vol. 114, no. 12, pp. 19291943, 2014.

[25] J. W. Eikelboom, S. S. Anand, S. R. Mehta, J. I. Weitz, C. Yi, and S. Yusuf, "Prognostic significance of thrombocytopenia during hirudin and heparin therapy in acute coronary syndrome without ST elevation: Organization to Assess Strategies for Ischemic Syndromes (OASIS-2) study," Circulation, vol. 103, no. 5, pp. 643-650, 2001. 